\title{
Sonographically Guided Patellar Tendon Fenestration
}

\section{Prognostic Value of Preprocedure Sonographic Findings}

\author{
Yassine Kanaan, MD, Jon A. Jacobson, MD, David Jamadar, MBBS, Jeffrey Housner, MD, \\ Elaine M. Caoili, MD
}

Received August 29, 2012, from the Department of Radiology, Texas Scottish Rite Hospital for Children, Dallas, Texas USA (Y.K.); and Departments of Radiology (J.A.J., D.J., E.M.C.) and Orthopedic Surgery (J.H.), University of Michigan, Ann Arbor, Michigan USA. Revision requested September 18, 2012. Revised manuscript accepted for publication October 10, 2012.

Address correspondence to Jon A. Jacobson, MD, Department of Radiology, University of Michigan, 1500 E Medical Center Dr, TC2910L, Ann Arbor, MI 48109-5326 USA.

E-mail: jjacobsn@umich.edu
Objectives - The purpose of this study was to determine whether any sonographic features of jumper's knee can predict the outcome after sonographically guided percutaneous patellar tendon fenestration.

Methods_-Patients were identified between July 2001 and March 2009 who had clinical and sonographic findings of jumper's knee, who had failed conservative treatment, and who had sonographically guided percutaneous fenestration of the patellar tendon. Pain levels were assessed before and 4 weeks after fenestration. Sonograms were retrospectively characterized with regard to echogenicity, size, definition, location, calcification, and hyperemia of the abnormal patellar tendon region. Sonographic findings before fenestration were correlated with clinical outcomes.

Results - A total of 45 patellar tendons (32 consecutive patients) were included in the study. The average prefenestration functional pain score was 3.6 (range, 2-5), and the average postprocedure functional pain score after 4 weeks was 1.4 (range, 0-5). The preprocedure sonographic finding that correlated with an improved clinical outcome after tendon fenestration was the presence of a well-defined tendon abnormality. No other sonographic findings were associated with the clinical outcome. After fenestration, 76\% (34 of 45) showed clinical improvement; 24\% (11 of 45) showed no change; and $0 \%$ ( 0 of 45$)$ had worse symptoms at 4 weeks.

Conclusions - The presence of a well-defined area of tendinosis on sonography was associated with clinical improvement after percutaneous sonographically guided fenestration of the patellar tendon. All tendons either improved (76\%) or showed no change $(24 \%)$ in the functional pain score at 4 weeks after fenestration.

Key Words - fenestration; patellar tendon; sonography; tenotomy

$\mathrm{P}$ atellar tendinosis or jumper's knee is an overuse injury associated with overloading of the knee extensor mechanism and characterized by pain and tenderness at the inferior pole of the patella. ${ }^{1,2}$ The result is mucoid degeneration and possible micro tears of the involved tendon with absence of inflammatory cells; therefore, the term "tendinosis" is a more accurate description of the resultant condition as opposed to "tendinitis." 3 Sonography has been used to diagnose jumper's knee where tendinosis appears as hypoechoic swelling of the tendon with possible increased flow on color and power Doppler imaging; coexisting interstitial tears appear as anechoic clefts. ${ }^{3}$ 
In addition to sonography's role in the diagnosis of tendon abnormalities, it has also been used to guide percutaneous tendon treatments. Tendon fenestration (dry needling or tenotomy) has been evaluated as a treatment option for chronic tendinosis of the Achilles tendon, common extensor tendon origin at the elbow, and others, including the patellar tendon. ${ }^{4}$ After percutaneous sonographically guided fenestration of the patellar tendon, excellent or good satisfaction scores have been reported in $81 \%$ of patients. ${ }^{5}$ However, identification of sonographic findings that would predict a positive outcome after sonographically guided fenestration would be helpful in the selection of patients who may be considered for this treatment.

In our clinical practice, we have noted variable appearances of patellar tendinosis on sonography with regard to echogenicity, size of the tendon abnormality, and vascularity on color and power Doppler imaging. It is unclear whether increased vascularity is beneficial when performing fenestration, as hemorrhage and growth factor release are proposed mechanisms for tendon healing. The purpose of this study was to determine whether sonographic features of jumper's knee predict the clinical outcome of sonographically guided patellar tendon fenestration.

\section{Materials and Methods}

\section{Patients}

Institutional Review Board approval was obtained, and informed consent was waived. Patients who had sonographically guided patellar tendon fenestration at the discretion of the sports medicine physician were identified from the radiology information system between July 2001 and March 2009. All patients had sonographically guided tendon fenestration as part of their routine clinical care if the following criteria were met: (1) history and physical examination findings were consistent with patellar tendinosis as determined by a fellowship-trained sports medicine physician; (2) sonographic findings showed tendinosis; and (3) the patient failed to respond to conservative treatment, including relative rest, pain medication, and physical therapy. Exclusion criteria were patients younger than 18 years, duration of symptoms less than 6 months, those who had prior patellar tendon surgery or fenestration, and patients with normal sonographic results. Of note, the patients in this study were part of a prior study that assessed patient outcomes after percutaneous sonographically guided fenestration. ${ }^{5}$ In contrast to that prior study, this research compared sonographic findings to a change in the functional pain score after fenestration.

\section{Clinical Examination}

All participants underwent a clinical knee examination as part of routine patient care by a fellowship-trained sports medicine physician with 13 years of experience. Additional imaging, such as magnetic resonance imaging and radiography, was also performed at the discretion of the sports medicine physician. Each patient was also assessed for the level of functional pain before tendon fenestration using the following classification: 0 , no pain; 1 , pain only after intense sports activity, no undue functional impairment; 2 , pain at the beginning and after sports activity, still able to perform at a satisfactory level; 3 , pain during sports activity, increasing difficulty in performing at a satisfactory level; 4, pain during sports activity, unable to participate in sports at a satisfactory level; and 5, pain during daily activity, unable to participate in sports at any level. ${ }^{6}$

\section{Sonographic Examination}

Sonography was performed by one of two fellowshiptrained musculoskeletal radiologists (each with $>12$ years of experience) using high-resolution sonography including color or power Doppler with 7-15-MHz linear transducers (HDI 5000 and iU22; Philips Healthcare, Bothell, WA). Liberal transmission gel was used in place of a standoff pad, and the patellar tendon was evaluated in both long and short axes.

\section{Fenestration Procedure}

For the percutaneous tendon fenestration procedure, needling was performed by the fellowship-trained sports medicine physician in conjunction with the fellowshiptrained musculoskeletal radiologist who provided sonographic guidance. A sterile field was set up and maintained throughout the procedure. A 27-gauge needle (Becton Dickinson and Company, Franklin Lakes, NJ) was first used to anesthetize the skin with a 1:1 mixture of $1 \%$ lidocaine to $0.25 \%$ bupivacaine. The syringe was then switched over to a 22-gauge needle (Becton Dickinson and Company) to perform the fenestration of the focal abnormalities within the tendon. The needle was guided from an inferior-to-superior approach, long axis to the patellar tendon, and in plane with the transducer and ultrasound beam. Approximately 20 to 30 needle passes were made through the abnormal region of the tendon with real-time sonographic imaging for continual guidance. Typically, initial passes through the focal abnormalities were resistant to needling, producing palpable crepitation, which eventually would resolve as the focal tendon abnormality softened. If the tendon abnormality included the bone-tendon interface, this area was specifically targeted as well. The 
procedure was determined to be complete when the needle had been passed through all abnormal regions of the tendon and enthesis at least once. In each case, the needle was advanced along the long axis of the transducer only when it was visualized sonographically to ensure accurate needle placement. Up to $3 \mathrm{~mL}$ of the lidocaine-bupivacaine mixture was injected into the tendon to maintain anesthesia as needed. If calcifications were identified on sonography, these areas were targeted with the same 22-gauge needle to disrupt and mechanically fragment the calcifications.

\section{Postfenestration Clinical Examination and Follow-up}

The postprocedure protocol consisted of relative rest until the first follow-up appointment at 4 weeks. Patients were allowed to continue usual activities of daily living during that period, with the addition of strengthening exercises beginning at 2 weeks after the procedure. All patients were evaluated at 4 weeks by the same fellowship-trained sports medicine physician, who recorded their functional pain measurement using the same classification scale as the preprocedure assessment.

\section{Retrospective Sonogram Interpretation}

Two musculoskeletal radiologists (each with $>12$ years of experience with musculoskeletal sonography) reviewed the sonograms retrospectively in consensus to characterize the patellar tendon abnormalities. The following data were recorded: length (centimeters), width (centimeters), anteroposterior dimension (centimeters), definition (well defined or poorly defined) of the focal tendinosis (hypoechoic abnormality), presence or absence of an interstitial tendon tear (anechoic clefts), ratio of the abnormal tendon echogenicity to the entire thickness of the tendon measured in the anteroposterior dimension, distance of the focal tendinosis from the patella, patellar cortical irregularity, and presence of calcification within the patellar tendon (echogenic foci with possible shadowing). The presence and location of increased vascularity on color or power Doppler imaging (if performed) were also evaluated, and the degree of increased vascularity was graded as high ( $>50 \%$ of the abnormal tendon area) or intermediate $(<50 \%$ of the abnormal tendon area). Both radiologists were blinded to the clinical outcome and all patient information. Patient medical records were also reviewed.

\section{Analysis}

A 1-way analysis of variance model was used to evaluate the continuous variables to determine whether there were any significant differences among the functional pain scores. The Fisher exact test was used to determine whether the presence of specific imaging features was associated with preprocedure and postprocedure functional pain scores. For all analyses, $P<.05$ was considered to indicate statistical significance.

\section{Results}

A total of 45 patellar tendons ( 32 consecutive patients) were included in the study. There were 6 female and 26 male patients with an age range of 19 to 51 years (average, 26 years). The average prefenestration functional pain score was 3.6 (range, $2-5$ ), comprising $0 \%$ (0 of 45 ) of patellar tendons with a score of 0 or $1,4 \%$ ( 2 of 45$)$ with a score of $2,42 \%$ ( 19 of 45$)$ with a score of $3,47 \%$ (21 of 45$)$ with a score of 4 , and $7 \%$ ( 3 of 45 ) with a score of 5 . There were no immediate complications after the tendon fenestration procedure or at 4 weeks after the procedure.

With regard to the preprocedure sonographic findings (Figures 1 and 2), tendinosis measured an average of $13.2 \mathrm{~mm}$ (range, $1.3-25 \mathrm{~mm}$ ) in the craniocaudal dimension and an average of $9.6 \mathrm{~mm}$ (range, $1.5-18 \mathrm{~mm}$ ) in the transverse dimension (mediolateral). The average tendon abnormality in the anteroposterior dimension was $2.7 \mathrm{~mm}$ (range, 2.1-9.3 mm). The ratio of the tendon abnormality in the anteroposterior dimension to the overall anteroposterior tendon thickness was $72 \%$ (range, 39\%-88\%). The tendon abnormality was well defined in $80 \%$ ( 36 of 45; Figure 1) and poorly defined in $20 \%$ (9 of 45). Interstitial tears were present in 18\% ( 8 of 45 ; Figure 2). The average distance of tendinosis from the patella was $0.6 \mathrm{~mm}$ (range, $0-10 \mathrm{~mm}$ ) with cortical irregularity of the patella present in $78 \%$ ( 35 of 45 ). Calcification was present within the area of tendinosis in $31 \%$ ( 14 of 45 ). Color or power Doppler imaging was used in 29 cases, which showed increased vascularity within the abnormal tendon in 52\% (15 of 29) of the cases (Figure 1): high vascularity in 2 cases and intermediate in 13 cases. Of these 29 tendons, 34\% (10 of 29) showed increased vascularity around the tendinosis ( 9 intermediate and 1 high vascularity).

The average 4-week postfenestration functional pain score was 1.4 (range, $0-5$ ), comprising 31\% (14 of 45) patellar tendons with a score of 0 (Figure 1$), 36 \%(16$ of 45 ) with a score of $1,4 \%$ ( 2 of 45$)$ with a score of $2,16 \%$ ( 7 of 45 ) with a score of $3,9 \%$ ( 4 of 45$)$ with a score of 4 , and $4 \%$ ( 2 of 45 ) with a score of 5 . After fenestration, 76\% (34 of 45) showed an improved functional pain score (Figure 3). The average postfenestration functional pain score of 1.4 decreased from the average preprocedure score of 3.6. For the interval change in each individual functional pain score (Figure 3), 24\% (11 of 45) of the patellar tendons had no 
change; $7 \%$ ( 3 of 45 ) had a change of 1 scores; $27 \%$ (12 of $45)$ had a change of 2 scores; $22 \%$ ( 10 of 45 ) had a change of 3 scores; $20 \%$ ( 9 of 45 ) had a change of 4 scores; and $0 \%$ ( 0 of 45 ) had a change of 5 scores. Of note, any change in the functional pain score was associated with an improvement rather than a detriment. One sonographic finding, the presence of a well-defined hypoechoic tendon abnormality, showed a statistically significant association with an improved clinical outcome, as indicated by an interval decrease in the functional pain score $(P=.02)$. Of the 36 patellar tendons that showed well-defined tendinosis, $81 \%$ ( 29 of 36) showed improvement in the pain score after sonographically guided fenestration. All other sonographic findings did not significantly correlate with an improved clinical outcome at 4 weeks after fenestration (interstitial tear, $P=.68$; cortical irregularity, $P=.51$; calcification, $P=.25$; and vascularity, $P=.84)$.

\section{Discussion}

Sonographically guided tendon fenestration has been investigated as a treatment option for jumper's knee. ${ }^{4,5}$ Our results show that patients with well-defined tendinosis of the patellar tendon on sonography showed significant

Figure 1. Images from a 21-year-old woman with jumper's knee who had improvement of her functional pain score from 4 to 0 after fenestration. Long-axis (A) and short-axis (B) sonograms of the proximal patellar tendon show well-defined hypoechoic tendinosis (arrows). Arrowheads indicate patellar tendon; and P, patella. Color Doppler sonogram (C) shows increased flow. Long-axis sonogram (D) shows the needle (curved arrows) during tenotomy.

A

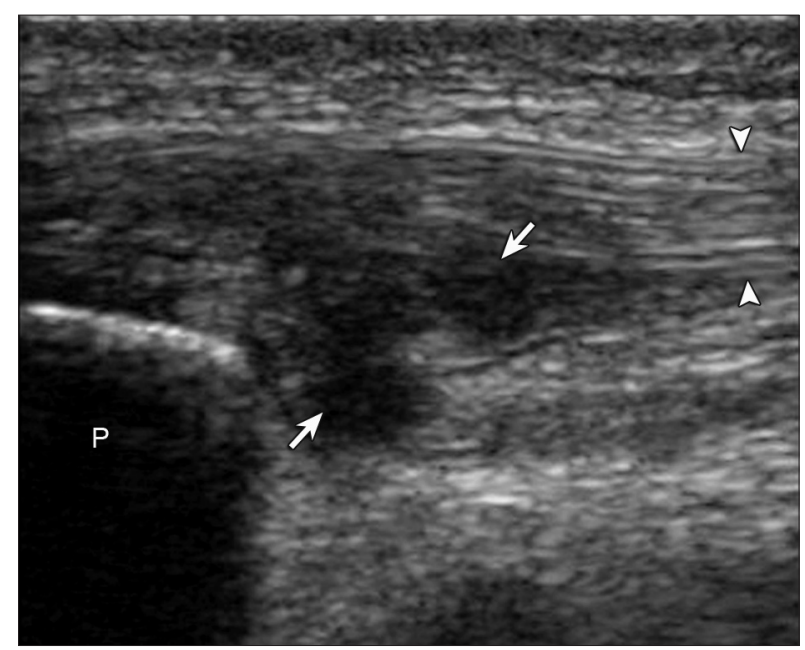

C

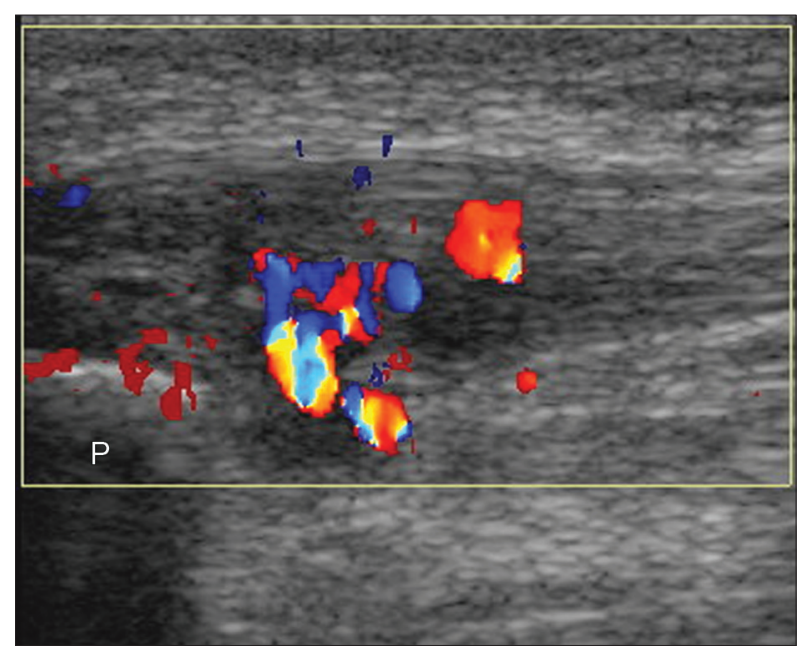

B

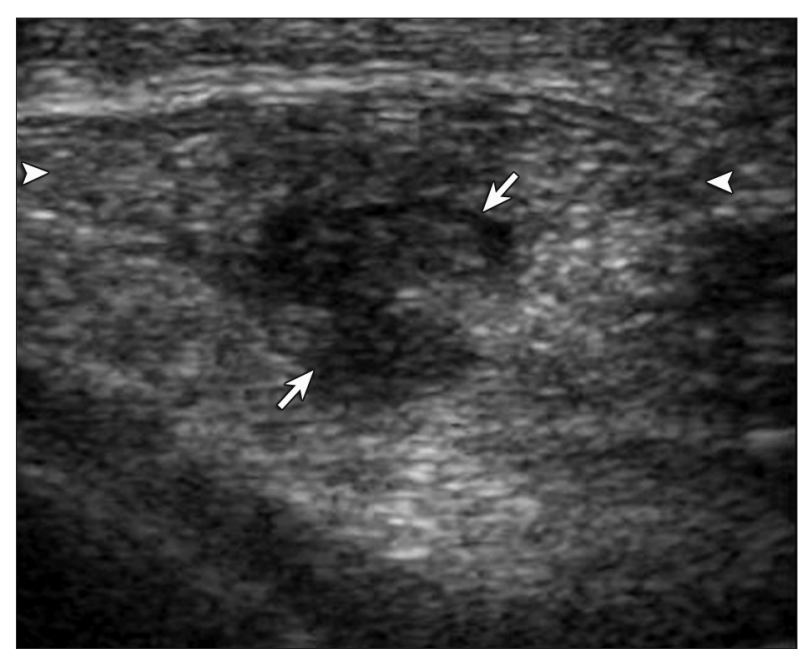

D

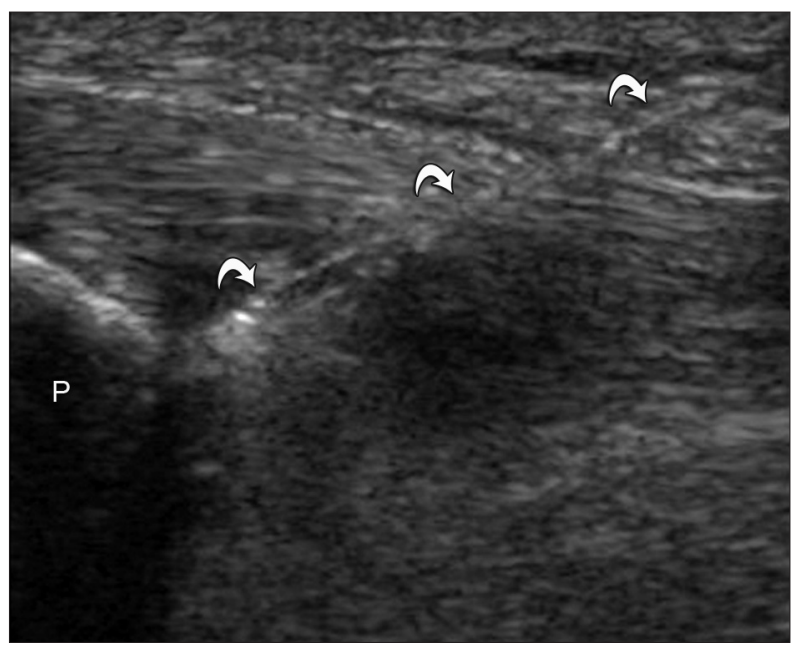


clinical improvement 4 weeks after sonographically guided tendon fenestration. All other sonographic findings, including hyperemia, were not significantly associated with the outcome after fenestration. After fenestration, 76\% (34 of 45) of patellar tendons showed clinical improvement; $24 \%$ (11 of 45) showed no change; and in no cases did symptoms become worse.

Patellar tendinosis or jumper's knee is clinically characterized by activity-related pain and soreness of the patellar tendon at the inferior pole of the patella. This condition is likely associated with overloading of the knee extensor mechanism and is a common overuse injury in professional athletes, especially those engaged in explosive jumping sports. ${ }^{6}$ In athletic populations, the estimated incidence of jumper's knee is between $13 \%$ and $20 \%{ }^{7,8}$ Patellar tendinosis may be difficult to treat and can be a source of chronic symptoms. ${ }^{9}$

Figure 2. Images from a 51-year-old man with jumper's knee who had improvement of his functional pain score from 3 to 1 after fenestration. Long-axis (A) and short-axis (B) sonograms of the proximal patellar tendon show a well-defined anechoic region interpreted as an interstitial tear (arrows) with surrounding hypoechoic tendinosis. Arrowheads indicate patellar tendon; and P, patella.

A

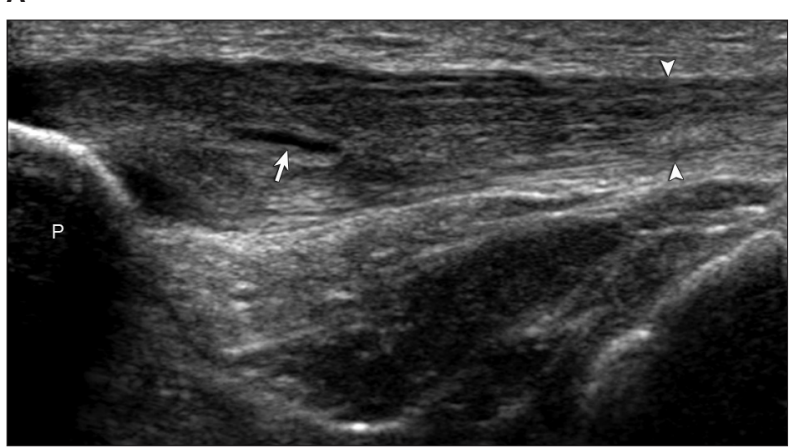

B

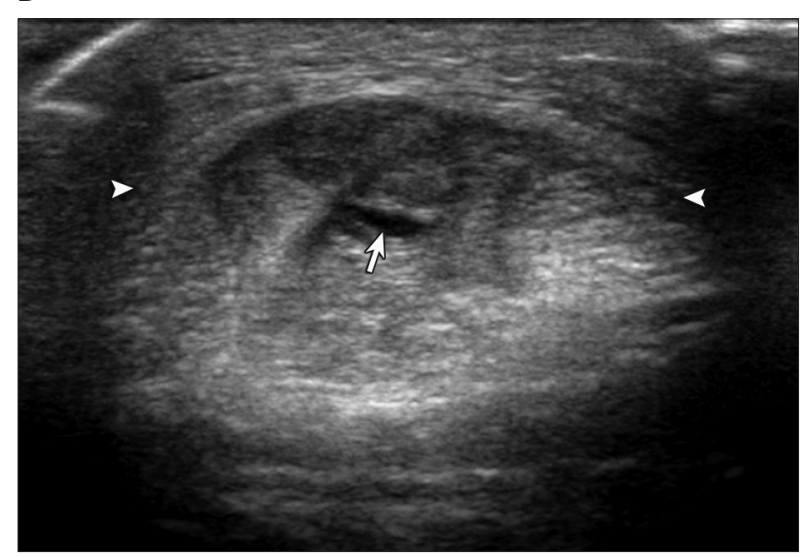

The injection therapies that have been used to treat patellar tendinosis have a similar fundamental principal of intentionally changing a chronic injury into an acutely injured state in the hope of inducing a high potential for healing. ${ }^{10}$ The mechanism of the healing process is not well understood; therefore, many theories are proposed. One hypothesis suggests that disruption of the fibers within the focal tendinosis causes hemorrhage and a subsequent inflammatory response, which incites the formation of granulation tissue that strengthens the tendon. ${ }^{11}$ Some investigators have found that preparations rich in growth factors, such as a platelet-rich clot, induce cell proliferation and synthesis of angiogenic factors in vitro. ${ }^{12}$ Others have theorized that basic fibroblast growth factor and transforming growth factor $\beta$ may act as humoral mediators in the stimulation of the healing process. ${ }^{13}$

Although there are data that support the benefit of tendon fenestration or dry needling alone in the treatment of tendinosis, there are other injection therapies and surgical options available. One such injection therapy involves the injection of a sclerosing agent, polidocanol, which is used to target and obliterate neovascularity. ${ }^{14-16}$ Injections of growth factors via autologous whole blood or plateletrich plasma have also been suggested as minimally invasive therapies that promote healing. . $^{10,17-22}$ Prolotherapy involves injection of an irritant (eg, hyperosmolar dextrose) that causes a local inflammatory response and up-regulation of growth factor expression, which results in collagen synthesis and a stronger tendon. ${ }^{23}$ Most of these injection therapies and dry-needling procedures described in the literature have been performed with the use of sonographic guidance because sonography can localize the tendon abnormality and accurately guide needle placement.

Figure 3. Change in functional pain scores. A higher score indicates more severe symptoms.

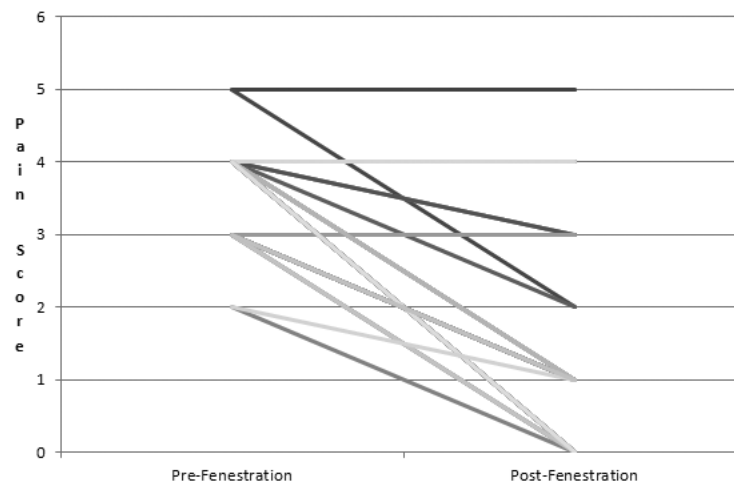


The surgical options for treatment of jumper's knee include excision of tendinotic tissue and curettage or drilling of the inferior patellar pole. Some less common procedures involve excision of the inferior patella and percutaneous longitudinal incisions of the patellar tendon..$^{24,25}$

The sonographic features of jumper's knee are wellestablished. ${ }^{3}$ Tendinosis appears as abnormal hypoechogenicity of the tendon with variable flow on color or power Doppler imaging, whereas interstitial tears appear as anechoic clefts. ${ }^{3}$ Involvement of the posterior aspect of the proximal patellar tendon is common; hyperechoic and shadowing calcifications and adjacent patellar cortical irregularity may be seen. With regard to the sonographic findings of jumper's knee in our study, patients with a welldefined margin of the patellar tendon abnormality had a statistically significant correlation with a positive clinical response to sonographically guided tendon fenestration at 4 weeks, indicated by an improved functional pain score. The other sonographic variables related to grayscale and color or power Doppler imaging did not show a statistical correlation with a clinical outcome.

With regard to increased blood flow in the setting of tendinosis, several investigations have shown that increased vascularity represents neovascularity, which correlates with symptoms when evaluating the Achilles and patellar tendons. ${ }^{15,26-28}$ In addition, sonographically guided sclerosis of the patellar tendon neovascularity at the ventral surface of the patellar tendon with polidocanol has shown to improve patient symptoms with moderate improvement in knee function. ${ }^{29}$ One of our hypotheses was that abnormal patellar tendons with more neovascularity would be associated with clinical improvement after tendon fenestration, as the neovascularity would be an ideal source for hemorrhage and intrinsic growth factors; however, that was not the case in our study. Of note, it has been shown that the presence of neovascularity of the Achilles tendon does not predict the clinical outcome after conservative treatment, unlike the presence of tendon inhomogeneity, which was associated with a poor outcome. ${ }^{28}$

With regard to the other sonographic variables that were assessed, we also hypothesized that a larger area of tendinosis or an area with interstitial tears would show less clinical improvement; however, that was not the case. One explanation for the lack of significance between most of the sonographic findings of jumper's knee and the clinical outcome is the fact that $76 \%$ of patients improved 4 weeks after the procedure, and no patients had worsening of symptoms. Further studies are needed with a larger sample size to address this possible explanation.
We acknowledge several limitations of this study. A selection bias may have been introduced because only patients who failed conservative treatment were included. Retrospective review of the sonograms may have also introduced error; however, videos or cine clips were recorded during each examination and were used as part of the retrospective review process, which was completed by two radiologists blinded to clinical data. In addition, longterm clinical follow-up assessment comparing prefenestration and postfenestration findings was not completed. Last, there was the lack of a control group or comparison treatment group, such as autologous blood or plateletrich plasma injection.

In conclusion, the presence of a well-defined area of tendinosis of the patellar tendon correlated significantly with clinical improvement of patients with jumper's knee 4 weeks after sonographically guided patellar tendon fenestration. Other sonographic findings, such as increased vascularity, did not correlate with clinical improvement. All patients treated with sonographically guided percutaneous patellar tendon fenestration either improved (76\%) or showed no change (24\%) in their functional pain scores.

\section{References}

1. Ferretti A, Puddu G, Mariani PP, Neri M. The natural history of jumper's knee: patellar or quadriceps tendonitis. Int Orthop 1985; 8:239-242.

2. Kujala UM, Aalto T, Osterman K, Dahlstrom S. The effect of volleyball playing on the knee extensor mechanism. AmJ Sports Med 1989; 17:766769.

3. Khan KM, Bonar F, Desmond PM, et al. Patellar tendinosis (jumper's knee): findings at histopathologic examination, US, and MR imaging. Victorian Institute of Sport Tendon Study Group. Radiology 1996; 200: 821-827.

4. HousnerJA,Jacobson JA, Misko R. Sonographically guided percutaneous needle tenotomy for the treatment of chronic tendinosis. J Ultrasound Med 2009; 28:1187-1192.

5. Housner JA, Jacobson JA, Morag Y, Pujalte GG, Northway RM, Boon TA. Should ultrasound-guided needle fenestration be considered as a treatment option for recalcitrant patellar tendinopathy? A retrospective study of 47 cases. Clin J Sport Med 2010; 20:488-490.

6. Blazina ME, Kerlan RK, Jobe FW, Carter VS, Carlson GJ.Jumper's knee. Orthop Clin North Am 1973; 4:665-678.

7. Jarvinen M. Epidemiology of tendon injuries in sports. Clin Sports Med 1992; 11:493-504.

8. Kujala UM, Friberg O, Aalto T, Kvist M, Osterman K. Lower limb asymmetry and patellofemoral joint incongruence in the etiology of knee exertion injuries in athletes. Int J Sports Med 1987; 8:214-220.

9. CookJL, Khan KM. What is the most appropriate treatment for patellar tendinopathy? Br J Sports Med 2001; 35:291-294. 
10. James SL, Ali K, Pocock C, et al. Ultrasound guided dry needling and autologous blood injection for patellar tendinosis. Br J Sports Med 2007; 41:518-522.

11. Edwards SG, Calandruccio JH. Autologous blood injections for refractory lateral epicondylitis. J Hand Surg Am 2003; 28:272-278.

12. Anitua E, Andía I, Sanchez M, et al. Autologous preparations rich in growth factors promote proliferation and induce VEGF and HGF production by human tendon cells in culture. J Orthop Res 2005; 23:281286.

13. Iwasaki M, Nakahara H, Nakata K, Nakase T, Kimura T, Ono K. Regulation of proliferation and osteochondrogenic differentiation of periosteum-derived cells by transforming growth factor-beta and basic fibroblast growth factor.J Bone Joint Surg Am 1995; 77:543-554

14. Hoksrud A, Ohberg L, Alfredson H, Bahr R. Ultrasound-guided sclerosis of neovessels in painful chronic patellar tendinopathy: a randomized controlled trial. Am J Sports Med 2006; 34:1738-1746.

15. Ohberg L, Alfredson H. Ultrasound guided sclerosis of neovessels in painful chronic Achilles tendinosis: pilot study of a new treatment. Br J Sports Med 2002; 36:173-177.

16. Ohberg L, Alfredson H. Sclerosing therapy in chronic Achilles tendon insertional pain: results of a pilot study. Knee Surg Sports Traumatol Arthrosc 2003; 11:339-343.

17. Hansen RB, Calandruccio JH, Kumar A. Autologous blood injection for the treatment of lateral epicondylitis. Paper presented at: 67th Annual Meeting of the American Academy of Orthopaedic Surgeons; March 15-19, 2000; San Francisco, CA.

18. Hildebrand KA, Woo SL, Smith DW, et al. The effects of platelet-derived growth factor-BB on healing of the rabbit medial collateral ligament: an in vivo study. Am J Sports Med 1998; 26:549-554.

19. Iyer VR, Eisen MB, Ross DT, et al. The transcriptional program in the response of human fibroblasts to serum. Science 1999; 283:83-87.

20. Knighton DR, Hunt TK, Thakral KK, Goodson WH III. Role of platelets and fibrin in the healing sequence: an in vivo study of angiogenesis and collagen synthesis. Ann Surg 1982; 196:379-388.

21. Mishra A, Woodall J Jr, Vieira A. Treatment of tendon and muscle using platelet-rich plasma. Clin Sports Med 2009; 28:113-125.

22. Mohle R, Green D, Moore MA, Nachman RL, Rafii S. Constitutive production and thrombin-induced release of vascular endothelial growth factor by human megakaryocytes and platelets. Proc Natl Acad Sci USA 1997; 94:663-668

23. Maxwell NJ, Ryan MB, Taunton JE, Gillies JH, Wong AD. Sonographically guided intratendinous injection of hyperosmolar dextrose to treat chronic tendinosis of the Achilles tendon: a pilot study. AJR Am J Roentgenol 2007; 189:W215-W220.

24. Karlsson J, Lundin O, Lossing IW, Peterson L. Partial rupture of the patellar ligament: results after operative treatment. Am J Sports Med 1991; 19:403-408.

25. Raatikainen T, Karpakka J, Puranen J, Orava S. Operative treatment of partial rupture of the patellar ligament: a study of 138 cases. Int J Sports Med 1994; 15:46-49.
26. Cook JL, Malliaras P, De Luca J, Ptasznik R, Morris M. Vascularity and pain in the patellar tendon of adult jumping athletes: a 5 month longitudinal study. BrJ Sports Med 2005; 39:458-461.

27. Malliaras P, Purdam C, Maffulli N, Cook J. Temporal sequence of greyscale ultrasound changes and their relationship with neovascularity and pain in the patellar tendon. BrJ Sports Med 2010; 44:944-947.

28. Zanetti M, MetzdorfA, Kundert HP, et al. Achilles tendons: clinical relevance of neovascularization diagnosed with power Doppler US. Radiology 2003; 227:556-560.

29. Hoksrud A, Torgalsen T, Harstad H, et al. Ultrasound-guided sclerosis of neovessels in patellar tendinopathy: a prospective study of 101 patients. Am J Sports Med 2012; 40:542-547. 